\title{
Mitigação de Ataques IDFs no Serviço de Agrupamento de Disseminação de Dados em Redes IoT Densas
}

\author{
Carlos Pedroso $^{1}$, Fernando Gielow ${ }^{1}$, Aldri Santos ${ }^{1}$, Michele Nogueira ${ }^{1}$ \\ ${ }^{1}$ Núcleo de Redes Sem-Fio e Redes Avançadas (NR2) - UFPR \\ Centro de Ciência de Segurança Computacional (CCSC) - UFPR \\ \{capjunior, fhgielow, aldri, michele\} @inf.ufpr.br
}

\begin{abstract}
The growth of IoT has made possible the creation of increasingly personalized services, among them, the industrial services, that often deal with massive amounts of data. However, as IoT grows, its threats are even greater. Among these threats, the false data injection attacks (FDI) stand out as being one of the most aggressive to data networks such as IoT. Although there are several mechanisms that deal with it they do not take into account the validation of the data, especially on the cluster data service. This work proposes an intrusion detection mechanism, called CONFINIT, against FDI attacks on the data dissemination service in IoT dense. It combines strategies of watchdog surveillance and collaborative consensus for the detection of attackers, guaranteeing the authenticity of the data collected by the devices. CONFINIT was evaluated in NS-3 and attained $99 \%$ of detection rate, 3.2\% of false negative and $3.6 \%$ of false positive rates, and increased by $30 \%$ the clustering without FDI attacks.
\end{abstract}

Resumo. O crescimento da IoT vem possibilitando a criação de serviços cada vez mais personalizados, entre eles destacam-se os serviços industriais que muitas vezes lidam com massiva quantidade de dados. Porém à medida que a IoT cresce as suas ameaças são ainda maiores. Entre as ameaças estão os ataques de injeção de dados falsos (IDF) que se destacam por serem um dos mais agressivos às redes de dados como a IoT. Embora existam mecanismos que lidam com essa ameaça, eles não consideram a validação dos dados, principalmente sobre o serviço de agrupamento de dados. Este trabalho propõe um mecanismo de detecção de intrusão contra ataques IDF, chamado CONFINIT, sobre o serviço de disseminação de dados em IoT densa. Ele combina estratégias de vigilância watchdog e consenso colaborativo para a detecção de atacantes, garantindo a autenticidade dos dados coletados pelos dispositivos. O CONFINIT foi avaliado no NS-3 e obteve $99 \%$ de taxa de detecção, 3,2\% de falsos negativos e 3,6\% de falsos positivos, e aumentou em até $30 \%$ os agrupamentos sem ataques IDF.

\section{Introdução}

A Internet das coisas (IoT) possibilita a conexão de diferentes tipos de objetos físicos, através de tecnologias como as redes de sensores sem fio (RSSF), RFID, GPS e NFC, entre outras. Os objetos que compõem a IoT possuem várias características como identidade, atributos físicos, heterogeneidade, e muitos deles usam interfaces inteligentes para estabelecerem comunicações entre si, além de apresentar alguma forma de mobilidade [Gubbi et al. 2013]. A IoT faz parte da evolução de domínios densos e complexos como 
processos industriais, logística, segurança pública e cidades inteligentes. Nesse contexto, o paradigma da Internet das coisas industriais (IIoT) vem recebendo maior atenção nos últimos anos. A IIoT trata da conexão de diferentes dispositivos dentro de uma indústria, possibilitando que todos trabalhem de forma sincronizada e organizada. Por tratar-se de uma variação da IoT, a IIoT ainda não está totalmente consolidada, apresentando diversas vulnerabilidades e desafios para sua solidificação [Mumtaz et al. 2017]. A IoT faz-se fundamental para coletar, disseminar e lidar com o volume de dados exigido por diversas aplicações nas suas tomadas de decisões [Minoli et al. 2017, Akpakwu et al. 2018].

Consequentemente, tratar e disseminar esse grande volume de dados resultante da interação entre diversos dispositivos expõe a IoT densa a diversas vulnerabilidades. Os ambientes IoT normalmente contam com dispositivos móveis e fixos e a infraestrutura varia conforme a interação. Parte dos dispositivos têm recursos limitados, pouca energia, baixa capacidade de processamento e armazenamento, além de sofrerem perdas nos links de conexão [Borgia 2014, Qiu et al. 2018]. Logo, a IoT torna-se alvo de inúmeras ameaças que violam atributos de segurança como, integridade, autenticidade e disponibilidade de serviços, entre eles a disseminação de dados dentro da rede [Yaqoob et al. 2017, Kouicem et al. 2018], o que prejudica a operação de diversas aplicações [Miorandi et al. 2012, Mendez et al. 2017].

Particularmente, entre as ameaças internas ao serviço de disseminação de dados na IoT, destaca-se o ataque de injeção de dados falsos (IDF), considerado um dos ataques de intrusão mais nocivo às redes de dados, devido à inconsistência das informações geradas e à imprevisibilidade do seu acontecimento [Sen and Madria 2017]. Em razão da sua complexidade, a detecção do ataque torna-se complexa e trabalhosa, pois normalmente os dispositivos maliciosos estão autenticados na redes e exercem suas funções padrão de coleta e disseminação de dados [Deng et al. 2016]. Os ataques podem ocorrer em diferentes períodos e de forma contínua, desorientando a rede. $\mathrm{O}$ ataque IDF pode ocorrer de duas formas, quando um dispositivo é capturado por outro e tem seus dados fabricados ou alterados, e quando o próprio dispositivo apresenta um comportamento de má conduta. Esse comportamento dificulta a identificação de dispositivos maliciosos e aumenta o tempo de mal funcionamento da rede, gerando inconsistência nos dados. Desta forma, torna-se imprescindível identificar e isolar os dispositivos maliciosos da rede.

Apesar de várias técnicas terem sido empregadas para tratar ataques IDF, seja no contexto de RSSF [Lu et al. 2012, Kumar and Pais 2018], Smart Grids [Li et al. 2017] ou IoT [Yang et al. 2017]; elas têm falhado ou não são adequadas ao contexto de IoT densa, visto que geram alto consumo de recursos, não checam os dados e poucas consideram a detecção colaborativa. Os esquemas de filtragem em rotas são constantemente aplicados em RSSF, usam a verificação de relatório em nós intermediários entre origem e destino, descartando pacotes com qualquer tipo de inconsistência. As técnicas de detecção colaborativas são alternativas para lidar com ataques IDF nas Smart Grids, onde cada dispositivo desempenha duas funções, as suas funções-padrão e a de agente colaborativo de detecção. Já os sistemas de detecção de intrusão (IDS) são alternativas robustas para lidar com os ataques em diferentes contextos, podendo ser baseados em dispositivos ou rede. Entretanto, eles também podem gerar alto consumo de recursos, além de gerar novas vulnerabilidades. Logo, torna-se fundamental para o desenvolvimento da IoT que os mecanismos sejam capazes de detectar e isolar a presença de ameaças de forma distribuída 
garantindo uma maior robustez para os serviço de agrupamento e disseminação de dados.

Este trabalho propõe um mecanismo para mitigação do ataque de injeção de dados falsos sobre o serviço de disseminação de dados de redes IoT. O mecanismo chamado CONFINIT (CONsensus Based Data FIlteriNg for IoT), busca detectar e isolar da rede IoT dispositivos maliciosos que apresentem comportamento malicioso ao serviço de agrupamento. O mecanismo utiliza agrupamentos por similaridade para lidar com densidade de dispositivos na rede. Ele combina as estratégias de watchdog para o monitoramento entre participantes e consenso colaborativo para a tomada de decisão sobre a presença de dispositivos maliciosos. Avaliação no simulador NS-3 mostrou que o CONFINIT alcança uma taxa de detecção de $99 \%$, até 3,2\% de falsos negativos e até 3,6\% de falsos positivos, e aumenta em até $30 \%$ o número de agrupamentos formados sem a presença de atacantes, e assim comprovando a sua eficácia.

O restante do artigo está apresentado da seguinte forma: a Seção 2 apresenta os trabalhos relacionados. A Seção 3 apresenta e descreve o funcionamento do CONFINIT. A Seção 4 descreve o método de avaliação e o desempenho do mecanismo juntamente com resultados obtidos. A Seção 5 conclui o artigo e apresenta direcionamentos futuros.

\section{Trabalhos Relacionados}

A demanda por um serviço de disseminação de dados seguro em redes densas na presença de diversas formas de ataques de intrusão tem sido o foco de diferentes trabalhos [Lu et al. 2012, Kumar et al. 2016, Yang et al. 2017, Cervantes et al. 2018, Bostami et al. 2019]. Em geral, o uso de Sistemas de Detecção de Intrusão (IDS) viabiliza identificar, localizar e mitigar comportamentos de má conduta de diversas naturezas [Kumar et al. 2016]. Particularmente, os ataques de IDF sobre o serviço de disseminação de dados nas IoT densas tornam-se danosos à rede devido ao grande volume de dados gerados pelos dispositivos e da sua importância à tomada de decisões pelas aplicações.

Em [Yang et al. 2017], os autores propuseram um IDS baseado em detecção de anomalias pelo uso de watchdog para mitigação do ataque de injeção de dados falsos. Eles tentam predizer eventos naturais ao usar dados coletados de vigilância ambiental da IoT através de monitoramento Hierárquico Bayesiano Espacial-Temporal (HBT). Logo após, usa-se uma estratégia de decisão estatística em um teste de probabilidade sequencial para identificar atividades maliciosas. Entretanto, o modelo HBT apresenta alto consumo energético e o teste probabilístico, embora efetivo, sobrecarrega a rede, e não considera a validação dos dados. Em [Cervantes et al. 2018], os autores apresentam uma IDS para mitigação de ataques sinkhole e selective forwarding no roteamento de redes IoT densas, onde a rede organizada em clusters e classifica os nós em três categorias, nó líder, associado e membro. Uma estratégia watchdog em níveis, monitora a relação entre encaminhamento e dados recebidos, definindo quais nós apresentam comportamento malicioso. Apesar disso, computar a confiança entre participantes com base na quantidade de dados recebidos e transmitidos não exclui a possibilidade do nó alterar os dados coletados. Um método frequentemente usado contra os ataques IDF em RSSF são os esquemas de filtragem em rota [Lu et al. 2012, Yu and Guan 2010, Wang et al. 2014]. Em [Lu et al. 2012], os autores propõem um esquema de autenticação cooperativa para filtrar dados falsos em RSSF, onde cada nó requer um número fixo de vizinhos para gerenciar a autenticação, que se baseia no roteamento de vizinhos. Os nós são autenticados de forma distribuída 
ao longo do roteamento dos dados até a estação base. O esquema adota a técnica de bit comprimido visando não sobrecarregar o canal, tornando-o adequado para filtrar dados injetados já que a autenticação ocorre ponto-a-ponto.Além disso, o uso de um protocolo de roteamento evita que dispositivos maliciosos comprometam outros dispositivos. Contudo, a proposta não consideram a manipulação dos dados, além de não identificar os dispositivos comprometidos. Em [Yu and Guan 2010], os autores apresentam um esquema de filtragem dinâmica em rota para lidar com dados falsos e ataque DoS em RSSF. Cada nó tem um conjunto de chaves de autenticação usada para endossar relatórios. A autenticação é garantida por um grupo de nós escolhidos antes da rede iniciar. Cada nó oferece sua chave aos nós encaminhadores que devem divulgar suas chaves, permitindo a eles verificarem todos os relatórios. Porém, a solução não considera a manipulação dos dados, e emprega um custo adicional devido à constante troca de chaves entre os dispositivos.

Uma maneira de alcançar uma detecção colaborativa é através do uso de Consenso entre dispositivos de uma rede [Colistra et al. 2014]. Em [Toulouse et al. 2015], os autores propuseram um sistema distribuído para detecção de anomalias, baseado em um protocolo de consenso médio entre participantes. O sistema busca identificar anomalias que possam gerar ataques DDoS. Assim, ele realiza análises em cada ponto de coleta de dados usando um classificador Bayes. Ao final a análise ocorre de forma redundante, paralelo ao nível de cada ponto de coleta de dados, o que evita o ponto único de falhas. $\mathrm{O}$ uso do consenso de forma distribuída facilita a tomada de decisão colaborativa. Contudo, o custo computacional de comunicação entre os participantes tende a sobrecarregar a rede e diminuir a efetividade do sistema. Em [Kailkhura et al. 2015], os autores desenvolvem um algoritmo robusto de consenso apoiado em média ponderada distribuída. O algoritmo visa permitir uma adaptação às regras locais estipuladas pela rede, onde se desenvolveu uma técnica de aprendizagem para estimar parâmetros operacionais ou peso de cada nó. Assim, torna-se possível automatizar regras locais de fusão ou atualização para mitigar ataques. Ele atua em uma rede distribuída, mas os autores não consideram a dinamicidade como fator de impacto, o que facilita as ações maliciosas.

Assim, faz-se necessário o desenvolvimento de soluções capazes de atuar de forma distribuída e que sejam capazes de identificar e isolar a presença de dispositivos maliciosos em redes IoT densas. Essas soluções, ao preservar a segurança dos dispositivos, não devem interferir no seu funcionamento padrão. Além disso, deve-se atender normalmente as características de uma IoT densa.

\section{Um Mecanismo para Mitigação de Ataques IDFs}

Esta seção apresenta o sistema CONFINIT (CONsensus Based Data FIlteriNg for $\boldsymbol{I} o \boldsymbol{T}$ ) para mitigação de ataques de injeção de dados falsos sobre o serviço de disseminação de dados em rede IoT densa voltada ao ambiente da indústria 4.0. Ele baseia-se na vigilância (monitoramento Watchdog) entre participantes para detectar anomalias na rede e usa uma técnica de Consenso Colaborativo para tomada de decisão. O objetivo é garantir a autenticidade dos dados disseminados na rede IoT para apoiar a tomada de decisões das aplicações. O CONFINIT atua no serviço de agrupamento executando numa estrutura de rede IoT densa no contexto industrial, conforme ilustra a Figura 1 e descrito a seguir, composta por nós heterogêneos, que podem ou não apresentar mobilidade. Além disso, assume-se que a comunicação entre os agrupamentos ocorre através dos nós líderes. 

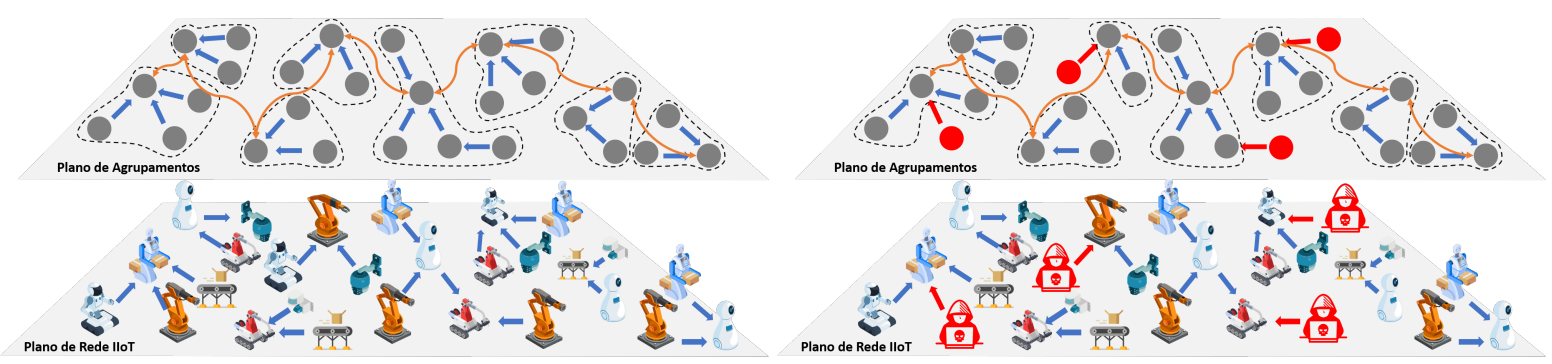

Figura 1. Modelos de agrupamentos de dados em rede loT e de ataque IDF

Considera-se uma rede IoT densa, como de um ambiente industrial, formada pelo conjunto $N$ de $n$ nós denotados por $N=\left\{n_{1}, n_{2}, n_{3}, \ldots, n_{n}\right\}$, onde $n_{i} \in N$. Cada nó $n_{i}$ tem um endereço físico exclusivo $(I d)$ que o identifica na rede. A comunicação ocorre através do meio sem fio mediante um canal assíncrono com perda de pacotes devido a ruídos e posição dos nós. Os nós diferenciam-se pelo nível de recursos que possuem, como energia, processamento e memória. Além disso, todos atuam na mesma faixa de transmissão para formarem os agrupamentos. Os nós começam como nós isolados buscando vizinhos para formarem os agrupamentos, podendo ou não participar de um agrupamento. Os nós que não participam de nenhum agrupamento podem ser detectados como atacantes ou suspeitos. Os nós podem assumir dois papéis: nó comum ou nó líder. Os nós comuns integram os agrupamentos enviando seus dados em direção aos líderes. Os líderes, por sua vez, são responsáveis por receber os dados e disponibilizá-los à aplicação.

A ameaça à rede se caracteriza como um ataque de injeção de dados falsos (IDF) onde o atacante, uma vez intruso na rede, apresenta má-conduta com características de adulteração, falsificação e fabricação de dados coletados por dispositivos IoT. O ataque IDF ocorre de duas formas. Na primeira forma, o atacante captura o dispositivo e manipula seus dados, seja alterando ou falsificando-os. Já na segunda, o próprio dispositivo é o atacante, e ele altera, fábrica ou manipula seus próprios dados. Considera-se que o ataque inicia-se por meio da exploração de vulnerabilidades decorrentes de outros ataques, além do atacante ter total conhecimento sobre a rede [Yang et al. 2017, Yaqoob et al. 2017].

\subsection{Arquitetura}

A arquitetura do CONFINIT é composta por dois módulos, Controle de Agrupamentos e Controle de Falhas como ilustrado na Figura 2. Ambos trabalham de maneira conjunta e em paralelo para garantir a disseminação de dados segura na rede. O módulo controle de agrupamentos organiza a rede em cluster e o módulo controle de falhas trata do monitoramento dos dispositivos e da detecção e isolamento de atacantes IDF.

O Módulo Controle de Agrupamentos (MCA) é responsável pela formação e manutenção dos agrupamentos dentro da rede. Ele avalia com base em um limiar de similaridade de leituras dos dispositivos (nós) que estão próximos e determina quando estão aptos para formar um agrupamento. Assim, ao receber uma mensagem ele verifica a identificação, a quantidade de vizinhos e as leituras desses vizinhos. O módulo (MCA) é compostos pelos componentes, Verificação de Similaridade (VA), Gerência de Agrupamento $(G A)$ e Disseminação de Leitura $(D L)$. O componente VA é responsável por receber e interpretar as mensagens trocadas entre os nós da rede. O componente GA trata de formar e manter os agrupamentos a partir da similaridade entre os nós, ele também é 
responsável pela eleição dos líderes. Já o componente DL é responsável por divulgar sua leitura, quantidade de leituras e vizinhos. Assim, todos os nós que receberem a mensagem saberão quando fazem ou não parte de um agrupamento.

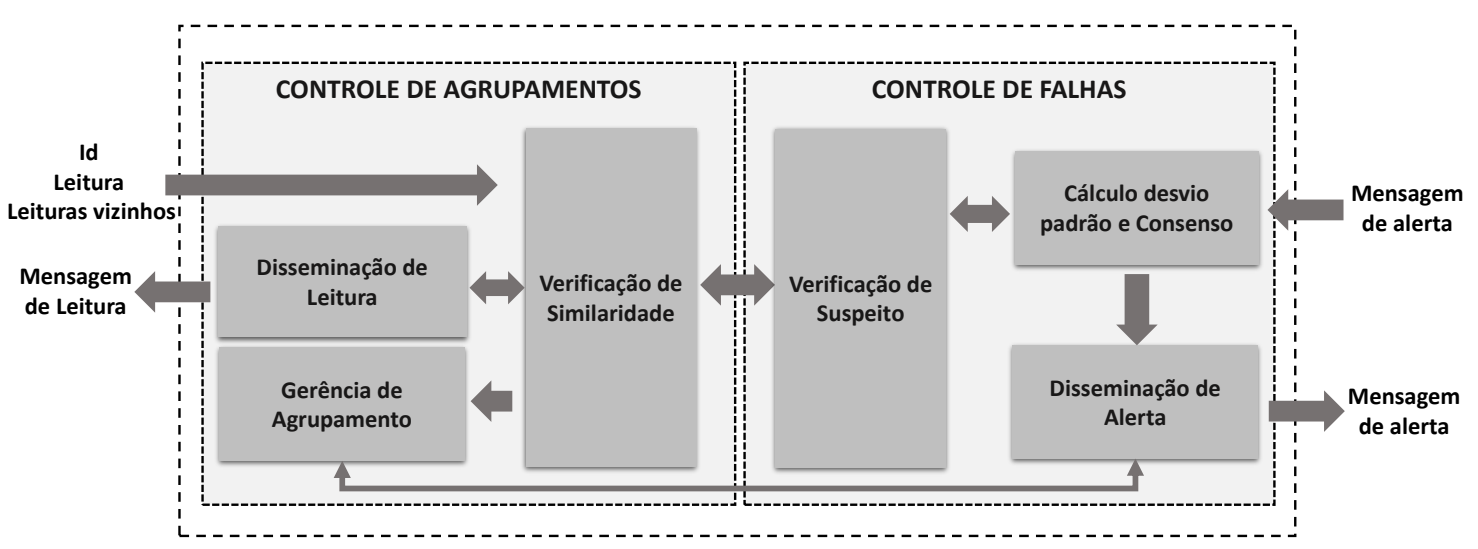

Figura 2. Arquitetura CONFINIT

O Módulo Controle de Falhas (MDF) garante a segurança da disseminação de dados entre os nós da rede IoT de modo que apenas leituras autênticas sejam disseminadas. Ele consiste dos componentes Verificação de Suspeito (VS), Detecção (DE) e Disseminação de Alerta (DA). O componente VS monitora os nós verificando aqueles que não respeitam o limiar de similaridade. O DE emprega a técnica de consenso colaborativo e desvio padrão para detectar os nós IDF. O consenso é a concordância e uniformidade de opiniões que os nós estabelecem por meio de troca de informações entre eles. O desvio padrão visa determinar quão discrepantes estão as leituras. O componente DA atua para isolar os nós atacantes e informar aos demais membros sobre esse ameaça. Logo, quando um ataque é detectado, os nós participantes da detecção propagam um alerta para que os líderes do agrupamento disseminem-o pela rede. Os dados propagados na mensagem de alerta consistem no $(I d)$ e leitura individual do atacante.

\subsubsection{Formação dos Agrupamentos}

Uma vez que o modelo de rede IoT é composto por uma grande diversidade de dispositivos com diferentes características, torna-se um desafio gerenciar e controlar esses dispositivos. Assim, o módulo Controle de Agrupamentos organiza a rede em grupos, com base nos líderes, para criar uma topologia de rede. Inicialmente, os nós começam suas operações de forma isolada, transmitindo e coletando mensagens de controle dos nós vizinhos para composição dos agrupamentos. O Algoritmo 1 apresenta o funcionamento do controle de agrupamentos. Periodicamente cada nó envia em broadcast uma mensagem de controle, informando seu identificador $(I d)$, sua leitura atual $L_{i n d}()$, a média de leitura agregada dos nós com leituras similares em sua vizinhança $L_{a g r}()$ e a quantidade de vizinhos $N_{v i z}()$ (l.1-l.5). O envio das mensagens leva em consideração um intervalo definido aleatoriamente a fim de evitar transmissões simultâneas entre todos os nós. Ao receber uma mensagem (l.6), o nó saberá a origem $\mathrm{org}$, a leitura individual $i R$ do nó emissor, a leitura agregada $a R$ de sua vizinhança e a quantidade de nós vizinhos considerados $n R$. Inicialmente, o nó atualiza as informações recebidas (l.7). As leituras agregadas médias são consideradas para verificar se a leitura do nó atual satisfaz ou não o limiar de similaridade (l.8). Assim, a verificação de similaridade é executada na (l.9). A vizinhança 
$N_{v i z}$ é atualizada definindo incluindo Org caso o limiar de similaridade seja respeitado (l.10) ou removendo o nó caso não (l.12). Esse processo ocorre dinamicamente em cada nó da rede, garantindo que todos possam manter sua estrutura de vizinhança atualizada.

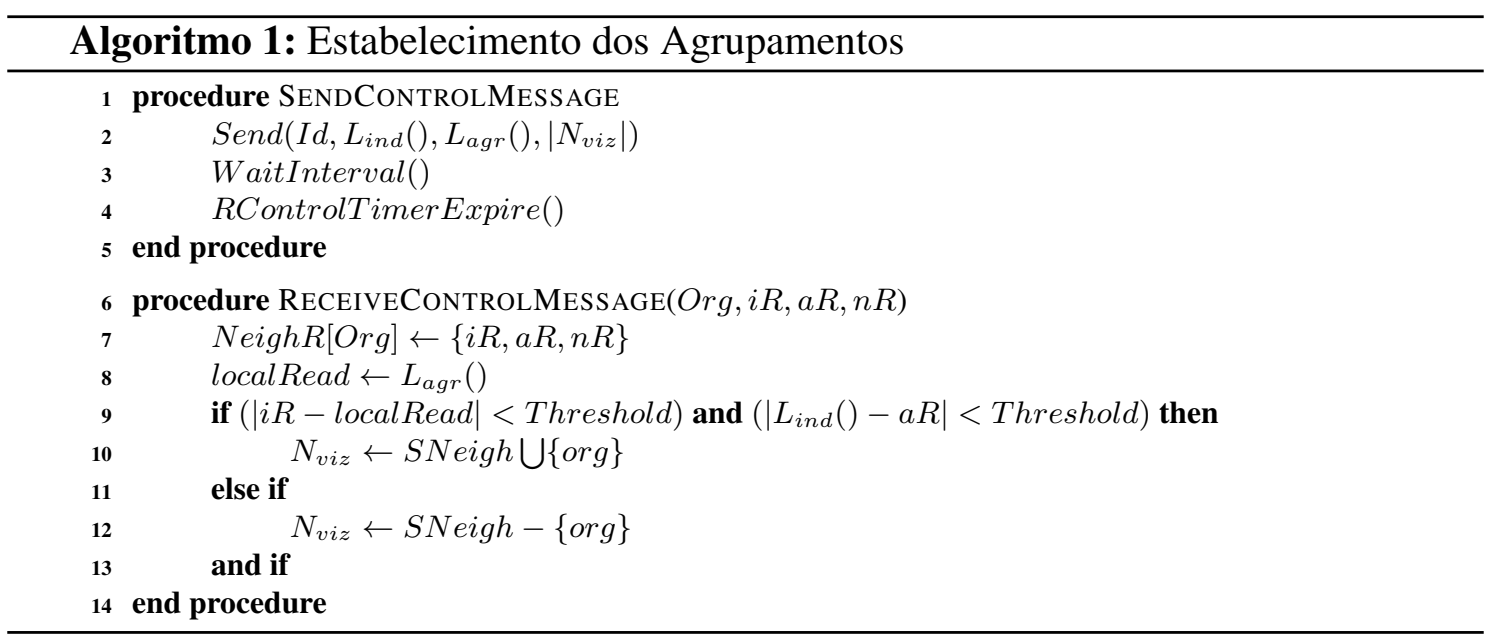

$$
\left|Y-\frac{X+\sum_{v \in \text { SNeigh }}(\text { NeighR }[v] . a R * N e i g h R[v] . n R)}{1+\sum_{v \in \text { SNeigh }}(N e i g h R[v] . n R)}\right|<C T h r e s h
$$

A relação de similaridade entre dois nós é calculada com base em seus valores de leitura, além de considerar o valor do limiar entre eles. A Equação 1 verifica a similaridade entre duas leituras, seguindo o modelo desenvolvido por [Gielow et al. 2015]. Em sua composição são atribuídos, as leituras dos nós, a quantidade de vizinhos e as leituras agregadas desses vizinhos, para determinar quando existe similaridade entre dois nós. Na equação, $X$ representa a leitura atual do nó e $Y$ a leitura com a qual ele está sendo comparada, a fim de verificar com base no limiar de similaridade se satisfazem essa diferença.

\subsubsection{Detecção de Falhas}

$\mathrm{Na}$ formação dos agrupamentos os nós que não atendem ao limiar de similaridade de leituras em um primeiro momento, são considerados suspeitos, passando a entregar uma lista de suspeitos. A utilização da lista se dá pelo fato de apresentar melhor avaliação sobre a detecção de falhas, já que os dispositivos em alguns momentos podem apresentar falhas, o que não caracteriza o comportamento de atacante. O Algoritmo2 detalha o funcionamento da detecção de falhas dentro da rede diante de uma ameaça IDF. A detecção passa a atuar efetivamente após a primeira troca de mensagens, visto que os nós necessitam de outras mensagens para fazer comparação. Assim, em um primeiro momento, é analisado se o nó em questão consta na lista de suspeito (l.8). Caso ele não esteja, mas suas leituras sejam consideradas suspeitas, ele é inserido na lista de suspeitos (l.12). Caso seja verificado que ele consta na lista de suspeitos, mas suas leituras respeitem o limiar definido, ele é removido da lista de suspeitos (l.14). 


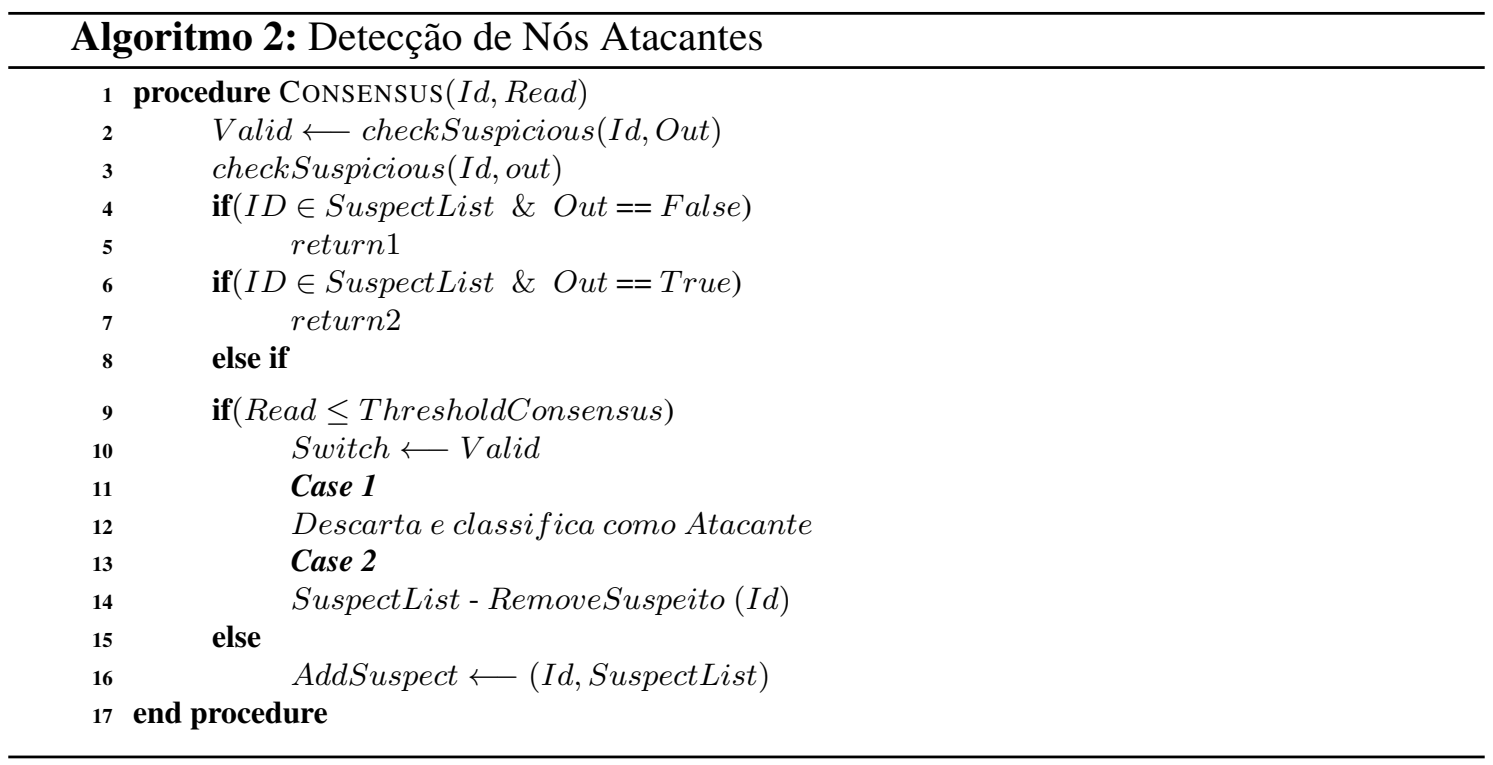

$$
D P=\sqrt{\frac{\sum_{i=1}^{n}\left(X_{i}-M_{A}\right)^{2}}{N}} \leq \text { Thresholconsensus }
$$

A Equação 2 determina o cálculo do consenso para verificar o desvio dos valores aferidos. Para tal, são usados os dados de leituras coletados dos participantes do consenso para comparação entre eles. Assim, utiliza-se um conjunto de dados $D=\left(x_{i}, x_{i+1}, \ldots, x_{n}\right)$, que representa as amostras a serem verificadas. O cômputo do consenso é indicado por $\sum_{i=1}^{n}$ que soma todos os valor do conjunto $D$, desde a primeira posição $(i=1)$ até a posição $n \in N$. O valor de $X_{i}$ é representado na posição $i$ no conjunto de dados. $M_{A}$ representa a média aritmética dos dados. $N$ representa a quantidade de dados a serem avaliados na formação do consenso. O Thresholdconsensus significa o valor predefinido que pode ser variado de acordo com o tipo de dados a serem avaliados.
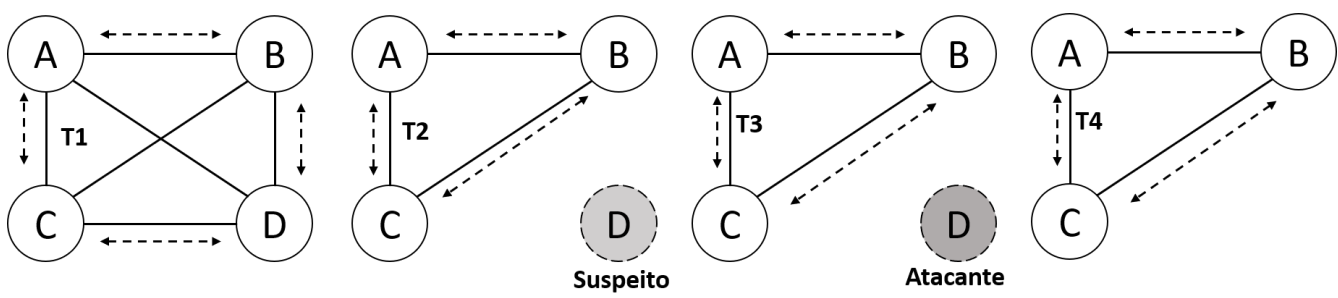

Figura 3. Formação de consenso entre os nós

A Figura 3 ilustra uma formação de consenso colaborativo entre os participantes para a detecção de falhas em razão de um atacante IDF. As setas pontilhadas representa a comunicação entre os nós $(\mathbf{A}, \mathbf{B}, \mathbf{C}, \mathbf{D})$ no instante $T l$, garantindo assim a troca de mensagem de controle entre eles. Já no instante $T 2$, apenas os nós $(\mathbf{A}, \mathbf{B}, \mathbf{C})$ agrupamse, visto que eles respeitam o limiar de similaridade. Entretanto, o nó $\mathbf{D}$ não respeita este limiar,então em um primeiro momento é classificado como suspeito. Em T3, o nó D envia novamente mensagens de controle para tentar fazer parte do agrupamento, assim o conjunto formado pelos nós (A, B e C) executam o cálculo da Equação 2, e classificam o 
nó $\mathbf{D}$ como atacante, já que ele novamente possui leituras distintas em relação ao conjunto. Por fim, em T4 apenas os nós honestos participam do agrupamento. Assim, a segurança é mantida pelos próprios participantes sem a necessidade de entidade externas.

\subsection{Funcionamento}

A formação de agrupamento ocorre de maneira dinâmica em cada nó da rede. As interações entre os nós realizam-se sob grandezas de espaço e tempo, assim as mensagens de dados são enviadas e recebidas pelos nós que estão dentro do raio de transmissão do emissor. Cada nó envia em broadcast, sua leitura, as leituras dos seus vizinhos, e a quantidade de vizinhos. Quando a mensagem é recebida, o nó receptor a interpreta, verificando seus campos e realizando o cálculo da similaridade. Se o limiar de similaridade é respeitado, o nó em questão passa a compor o agrupamento. Entretanto, se o limiar não for respeitado ele passa a integrar a lista de suspeito em um primeiro momento,

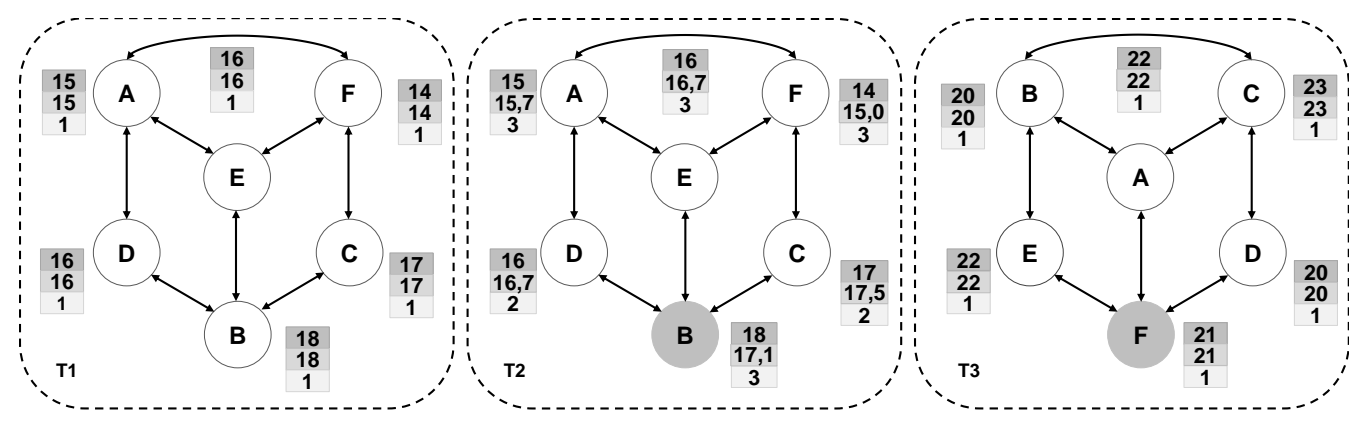

Figura 4. Formação dos agrupamentos

A Figura 4 ilustra um exemplo do funcionamento do mecanismo na formação dos agrupamentos e eleição dos líderes. As arestas sólidas indicam os nós que estão dentro do raio de transmissão um do outro e podem trocar mensagens. As caixas ao lado de cada nó correspondem à estrutura que indica, de cima para baixo, a leitura individual do nó, a leitura agregada sua e de seus vizinhos, e a quantidade de leituras agregadas. Assim, considera-se um limiar de similaridade $=3$ para formação dos agrupamentos. Cada instante $T$ corresponde a um troca de mensagem entre os nós para formar os agrupamentos e eleger os líderes. Com o controle de agrupamentos operando desta maneira, cada nó mantém atualizada suas informações através da troca de mensagens. Essa estrutura determina quais nós da vizinhança são vistos como membros do mesmo agrupamento, garante uma melhor escalabilidade à rede; além de ajudar a classificar nós com leituras divergentes, facilitando assim a identificação de atacantes pelo módulo de controle de falhas.

A detecção de falhas atua considerando a formação dos agrupamentos. Assim, os nós que não respeitam o limiar de similaridade passam a integrar uma lista de suspeitos em um primeiro momento. A classificação dos nós que não fizeram parte do agrupamento tem início na mensagem de controle, que sem os campos devidamente preenchidos são descartadas. Na formação dos agrupamentos, um nó que está próximo fisicamente de seus vizinhos em determinado instante e apresenta leituras distintas não respeitando o limiar de similaridade, pode ser considerado um nó suspeito em um primeiro momento. Assim, ele passa à integrar a lista de suspeitos, a qual contém nós que apresentaram um comportamento anômalo, mas não necessariamente são atacantes. Quando o nó em questão passa a integrar a lista de suspeitos e tenta participar do agrupamento, e novamente não 
consegue devido à leituras distintas, ele é classificado como atacante. Logo, seu $(I d)$ é inserido em uma lista que contém todos os $(I d s)$ das ameaças. Em seguida, o líder do agrupamento envia uma mensagem aos outros líderes avisando sobre a ameaça para que, caso ele tente se agrupar em outro momento, não consiga.

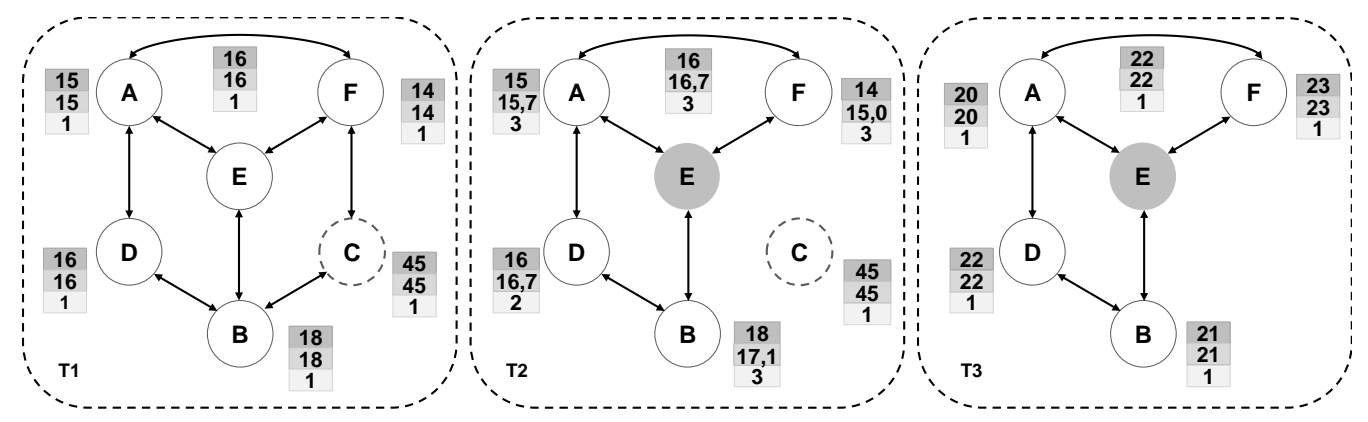

Figura 5. Detecção de falhas

A Figura 5 ilustra um exemplo da detecção de um ataque, onde cada instante $T$ corresponde à um processo de agrupamento e os nós que satisfazem o limiar de similaridade passam a formar um agrupamento. Assim, no instante $T 1$ os nós $(\mathbf{A}, \mathbf{B}, \mathbf{D}, \mathbf{E}$, F) têm seus valores de leitura individual variando entre 14 a 18, respeitando o limiar de similaridade entre eles. Entretanto, o nó $\mathbf{C}$ apresenta um valor de 45 para sua leitura, o que diverge muito em relação aos seus vizinhos espaciais, logo, não respeita o limiar de similaridade. Desta forma, o nó $\mathbf{C}$ não pode fazer parte do agrupamento nesse momento. No instante $T 2$ o nó $\mathbf{C}$ novamente tenta agrupar-se, mas como seu ( $I d)$ já consta na lista de suspeitos, é feito um consenso entre os participantes com base em suas leituras e comparadas às do nó $\mathbf{C}$. Logo, constata-se que $\mathbf{C}$ é um atacante, não podendo integrar nenhum agrupamento. No instante $T 3$, retirou-se o nó $\mathbf{C}$ da rede e é disseminada uma mensagem de alarme direcionada aos líderes com o $(I d)$ do atacante em questão.

\section{Avaliação}

Esta seção descreve a avaliação de desempenho do mecanismo CONFINIT. Ele foi implementado no simulador NS-3, versão 3.28. O simulador permite representar um ambiente de massiva escala de objetos IoT num cenário industrial com as características da rede. O cenário desenvolvido visa criar um ambiente próximo a de uma indústria, onde os dispositivos IoT estão sobre os objetos industriais representando o modelo de uma rede IIoT. Esses objetos podem variar conforme o tipo de indústria avaliada e seu tipo de função. O objetivo é usar leituras reais; assim, o ambiente de simulação foi baseado na coleta de dados de sensores de pressão de gás. Os dados usados foram coletadas e disponibilizados pelo laboratório UCI Machine Learning Repository [UCI 2013] e os datasets estão disponíveis no site para análises. Assim, busca-se chegar próximo de um ambiente real.

O cenário avaliado é composto por 100 nós distribuídos aleatoriamente em uma área retangular de $400 m \times 200 m$ operando por $1200 \mathrm{~s}$ com um raio de transmissão de $100 \mathrm{~m}$. A definição do raio de transmissão levou em conta a proporção da área de cobertura para ser definido e não comprometer os resultados obtidos, pois uma maior cobertura poderia interferir na formação dos agrupamentos e do consenso. A área definida busca representar um ambiente IoT denso, isso deve-se ao fato que os dados foram obtidos de um ambiente real, porém sem descrições sobre o tamanho da área. Assim, ela foi aferida com base nos 
trabalhos [Cervantes et al. 2018, Kumar and Pais 2018]. A comunicação entre os dispositivos ocorre através do protocolo IPv6, sendo estabelecida uma rede ad-hoc no padrão IEEE 802.15.4. Além disso, o protocolo de agrupamento DDFC [Gielow et al. 2015] foi implementado como parte do mecanismo para formação dos agrupamentos. Contudo, alterou-se o controle de mensagens, o intervalo adaptativo e a integração com IPv6 para melhor adaptá-lo ao contexto de rede IoT densa, visto que o DDFC foi proposto para outro cenário. Além disso, a avaliação do DDFC foi feita a fim de validar o impacto do ataque IDF em agrupamentos.

Tabela 1. Métricas de avaliação

\begin{tabular}{l|c}
\hline \hline Descrição & Equações \\
\hline $\begin{array}{l}\text { Taxa de detecção }\left(T_{d e t}\right) \text {, calcula os ataques IDF identificados correta- } \\
\text { mente pelo mecanismo a partir das interações entre os participantes. O } \\
\text { cálculo da }\left(T_{d e t}\right) \text { entende a razão entre o total de detecção }\left(\text { det }_{n i}\right), \text { e a } \\
\text { quantidade de ataques inseridos }\left(A_{\text {ins }}\right) .\end{array}$ & $\left(T_{d e t}\right)=\frac{\sum \text { det }_{n i}}{A_{\text {ins }}}$ \\
\hline $\begin{array}{l}\text { Taxa de Falsos positivos }\left(T_{f p}\right) \text { define a quantidade de vezes que o me- } \\
\text { canismo identificou um ataque IDF quando o mesmo não existia. Seu } \\
\text { cálculo ocorre através da divisão entre quantidade de detecção }\left(\text { det }_{n i}\right), \\
\text { pelo total de interações feitas à rede }\left(T_{i n t}\right) .\end{array}$ & $T_{f p}=\frac{\sum \text { det }_{n i}}{T_{i n t}}$ \\
\hline $\begin{array}{l}\text { Taxa de Falsos Negativos }\left(T_{f n}\right) \text { calcula o percentual de nós identifica- } \\
\text { dos de forma errada como não intrusos. O cálculo é representado por } X \\
\text { que é o total de interações, i.e. envio de mensagens de controle e }\left(T_{d e t}\right)\end{array}$ & $T_{f n}=|X|-T_{d e t}$ \\
a taxa de detecção do ataque. & \\
\hline \hline
\end{tabular}

O modelo de ataque utilizado baseia-se no modelo descrito em [Deng et al. 2016]. Nele o atacante busca alterar os dados de uma matriz de leituras de energia. Para isso dois tipos de dados são inseridos, um que visa uma pequena alteração e outro que altera completamente o dado coletado. Para melhor se adequar ao cenário desenvolvido, optou-se pela variação dos dados manipulados pelo atacante. Desta maneira, são selecionados nós específicos para atuarem como atacantes dentro da rede. Os atacantes têm total conhecimento sobre os dados trafegados na rede, facilitando assim sua interação com a rede na manipulação dos valores coletados. Assim, considera-se que os atacantes são capazes de manipular os dados seja, alterando, falsificando ou mesmo inserindo valores distintos aos coletados. Além disso, a atuação de nós suspeitos não determina que ele possa apresentar em um segundo momento comportamento de atacante. Desta forma, chegou-se o mais próximo ao comportamento real do ataque IDF. Os gráficos apresentados foram obtidos a partir de 35 simulações com um intervalo de confiança de 95\%. A Tabela 1 apresenta as métricas de avaliação usadas para mensurar o desempenho do CONFINIT na detecção do ataque IDF, e elas foram escolhidas com base em [Yang et al. 2017].

\subsection{Resultados}

A Figura 6 apresenta os desempenho do CONFINIT em relação a disponibilidade dos agrupamentos formados com base na quantidade de nós e atacantes presente na rede. Observar que o número de agrupamentos disponível varia conforme a relação de nós e atacantes presentes na rede. Em relação ao ataque IDF, observou-se que ele tem impacto direto na quantidade de agrupamentos formados. Entretanto, como esperado, $10 \%$ de ataques sobre a rede têm um impacto mais acentuado que 5\% e 2\%. Em alguns casos, o 
número de agrupamentos formados sem a presença do CONFINIT chegou a apresentar uma queda de até $35 \%$. Este comportamento afeta a quantidade de dados disponibilizados e consequentemente interferindo na tomada de decisões. O desempenho obtidos pelo mecanismo evidenciam sua eficiência em manter os agrupamentos seguros e disponíveis. A precisão na formação dos agrupamentos considera o fato do cenário ser estático, isto é, não sofrer com mudanças quanto à posição dos nós. Porém, as leituras apresentam uma variação quanto aos seu valores. Isto é evidente nas baixas variações no número de agrupamentos ao longo do tempo. Além disso, considera-se a quantidade não determinista de nós por agrupamentos, ou seja, não há um número fixo de integrantes por agrupamento.
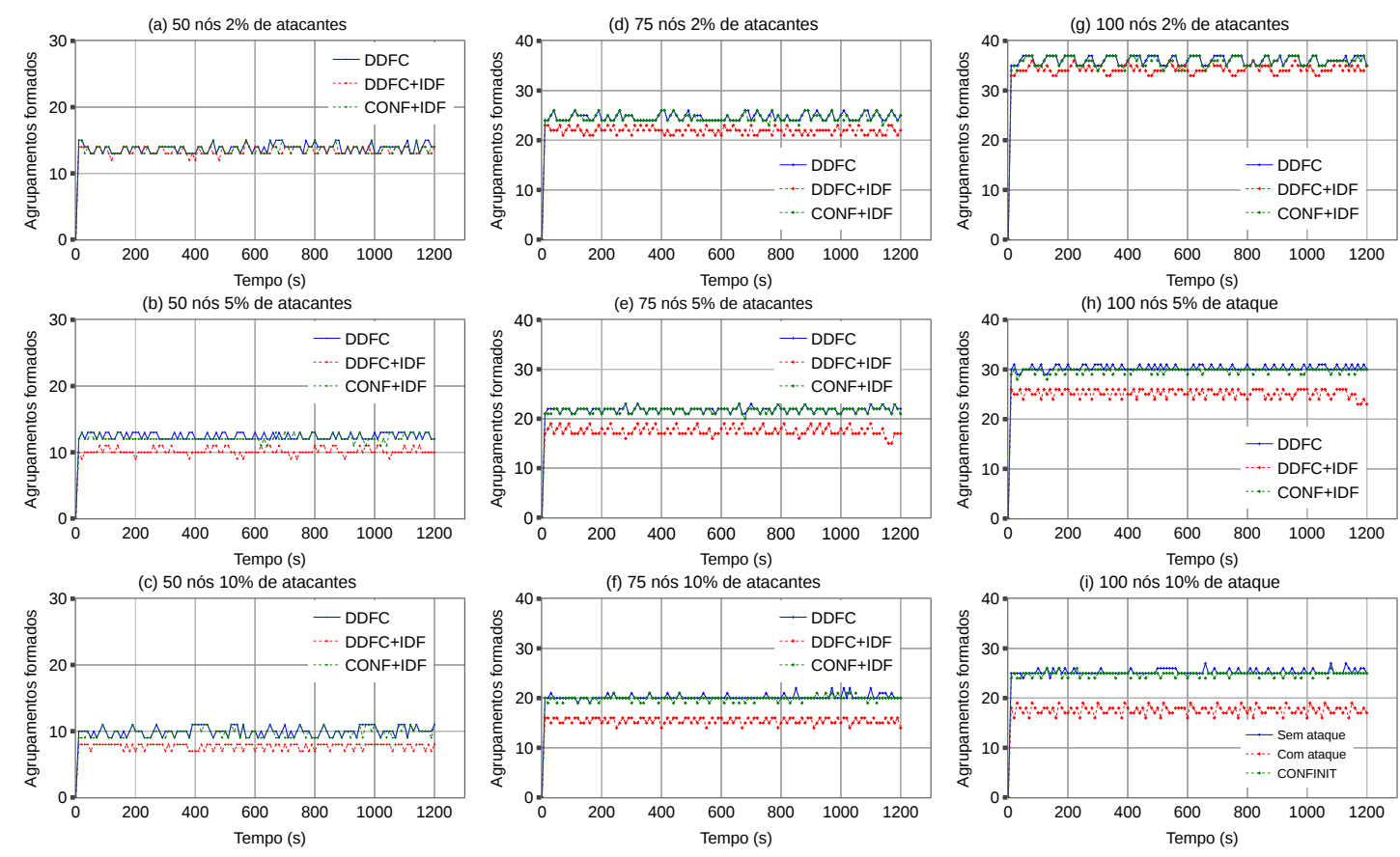

Figura 6. Número de agrupamentos formados ao longo do tempo

A Figura 7 (a) apresenta a capacidade de detecção e mitigação obtida pelo mecanismo CONFINIT sobre o ataque. O mecanismo obteve uma taxa média de detecção de $\left(T_{d e t}\right)$ 97\%, inclusive alcançando algumas vezes um taxa de média de $100 \%$ dependendo da variação no número de nós inseridos na rede. Em razão do comportamento dos dados apresentados, tem-se 95\% de certeza sobre eles. Esta efetividade na detecção deve-se à vigilância entre os participantes empregada pelo watchdog que avalia as mensagens trocadas; além disso, a formação de consenso colaborativo garante uma melhor validação e a alta taxa de detecção entre os nós. Também observa-se que a taxa de detecção possui pouca variação em relação à quantidade de nós na rede e a porcentagem de ataques inseridos, demonstrando a alta capacidade do mecanismo em lidar com um ambiente denso.

As Figuras (b) e (c) da Figura 7 apresentam o desempenho do CONFINIT em relação a taxas às de falsos negativos e falsos positivos na presença do ataque IDF. $\mathrm{O}$ CONFINIT obteve uma taxa média de falsos negativos variando de $2 \%$ para $2 \%$ de atacantes e 3,2\% para $10 \%$ de atacantes. A diferença média entre os cenários ficou em 1,2\%, representando uma baixa variação entre eles. Isso demonstra que poucos nós atacantes não são detectados pelo CONFINIT. A falha na detecção de um atacante pode acontecer quando há um erro no cálculo da similaridade, e o nó é indicado como suspeito; e portanto 

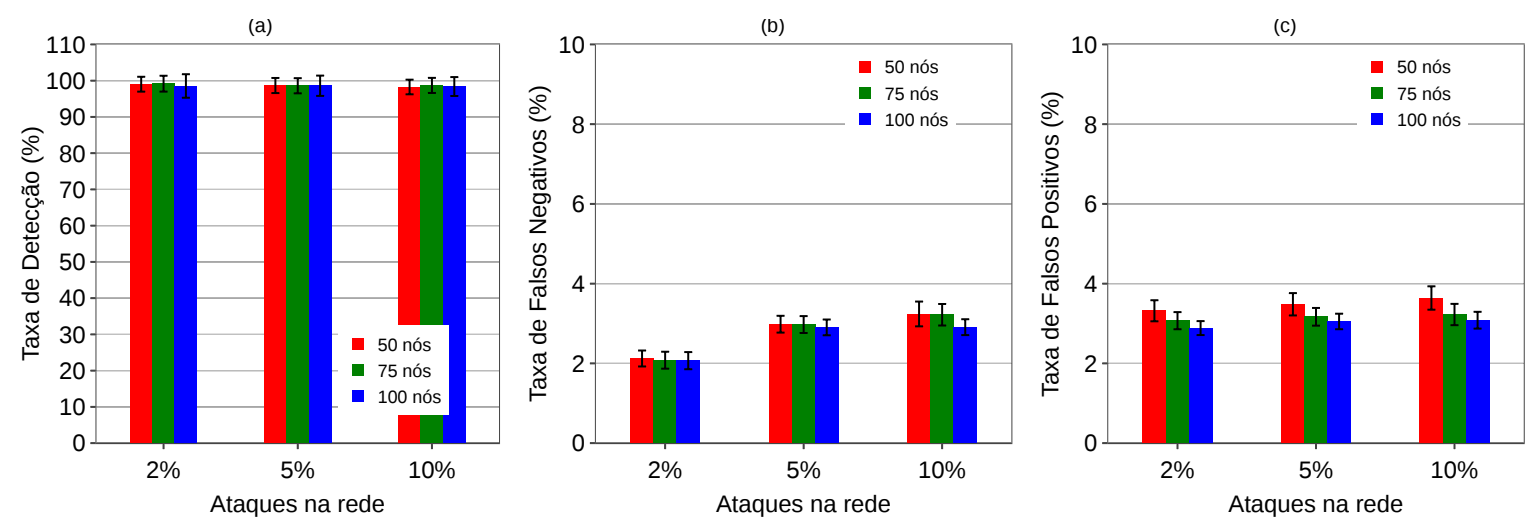

Figura 7. Taxas de detecção $\left(T_{d e t}\right)$ de falsos negativos $\left(T_{f n}\right)$ e falsos positivos $\left(T_{f p}\right)$

a fase de consenso acaba por identificar de maneira errada esse nó como atacante. Desta forma, os nós da rede podem demorar a identificar um atacante na rede. Com relação às taxas de falsos positivos, o CONFINIT obteve uma taxa média variando de $2,8 \%$ para $2 \%$ de atacantes e 3,6\% para $10 \%$ de atacantes. A diferença média entre os cenários ficou em $0,8 \%$, apresentando uma baixa variação entre eles. As detecções erradas também são decorrentes de erros no cálculo de consenso entre os nós monitores de um atacante, que pode apresentar baixo desvio de suas leituras. Logo, num primeiro momento eles são considerados suspeitos, porém conforme as novas interações e troca de mensagens entre os nós, os novos cálculos identificam os nós corretos.

\section{Conclusão}

Este trabalho apresentou o mecanismo CONFINIT para mitigação de ataque de injeção de dados falsos em rede IoT densa no contexto Industrial. Para tal, o mecanismo organiza a rede em agrupamentos para lidar com a densidade dos nós levando em consideração a similaridade de leituras entre eles. CONFINIT baseia-se em uso de watchdog e consenso colaborativo de modo a vigiar o comportamento dos nós com relação as suas informações de leitura, quantidade de vizinhos e leituras agregadas, a fim de determinar nós com um comportamento malicioso comparados aos outros. Os resultados demonstraram a eficácia do CONFINIT na detecção e mitigação de nós atacante IDF e a garantia de disponibilidade de apenas dados legítimos. Como trabalhos futuros pretende-se avaliar a eficiência do CONFINIT em diferentes contextos de redes IoT densa que também exijam agrupamentos de dados e apresentem outras variações de leituras, bem como uma avaliação do consumo de energia e da mobilidade na formação dos agrupamentos.

\section{Referências}

Akpakwu, G. A., Silva, B. J., Hancke, G. P., and Abu-Mahfouz, A. M. (2018). A survey on 5g networks for the internet of things: Communication technologies and challenges. IEEE Access, 6:3619-3647.

Borgia, E. (2014). The internet of things vision: Key features, applications and open issues. Computer Communications, 54:1-31.

Bostami, B., Ahmed, M., and Choudhury, S. (2019). False data injection attacks in internet of things. In Performability in Internet of Things, pages 47-58. Springer.

Cervantes, C., Nogueira, M., and Santos, A. (2018). Mitigação de ataques no roteamento em iot densa e móvel baseada em agrupamento e confiabilidade dos dispositivos. In Anais do XXXVI Simpósio Brasileiro de Redes de Computadores e Sistemas Distribuídos. SBC. 
Colistra, G., Pilloni, V., and Atzori, L. (2014). Task allocation in group of nodes in the iot: A consensus approach. In 2014 IEEE International Conference on Communications (ICC), pages 3848-3853. IEEE.

Deng, R., Xiao, G., Lu, R., Liang, H., and Vasilakos, A. V. (2016). False data injection on state estimation in power systems - attacks, impacts, and defense: A survey. IEEE Transactions on Industrial Informatics, 13(2):411-423.

Gielow, F., Jakllari, G., Nogueira, M., and Santos, A. (2015). Data similarity aware dynamic node clustering in wireless sensor networks. Ad Hoc Networks, 24:29-45.

Gubbi, J., Buyya, R., Marusic, S., and Palaniswami, M. (2013). Internet of things (iot): A vision, architectural elements, and future directions. Future generation computer systems, 29(7):1645-1660.

Kailkhura, B., Brahma, S., and Varshney, P. K. (2015). Consensus based detection in the presence of data falsification attacks. arXiv preprint arXiv:1504.03413.

Kouicem, D. E., Bouabdallah, A., and Lakhlef, H. (2018). Internet of things security: A top-down survey. Computer Networks.

Kumar, A. and Pais, A. R. (2018). Deterministic en-route filtering of false reports: A combinatorial design based approach. IEEE Access, 6:74494-74505.

Kumar, S. A., Vealey, T., and Srivastava, H. (2016). Security in internet of things: Challenges, solutions and future directions. In 49th HICSS, pages 5772-5781. IEEE.

Li, B., Lu, R., Wang, W., and Choo, K.-K. R. (2017). Distributed host-based collaborative detection for false data injection attacks in smart grid cyber-physical system. Journal of Parallel and Distributed Computing, 103:32-41.

Lu, R., Lin, X., Zhu, H., Liang, X., and Shen, X. (2012). Becan: A bandwidth-efficient cooperative authentication scheme for filtering injected false data in wireless sensor networks. IEEE transactions on parallel and distributed systems, 23(1):32-43.

Mendez, D. M., Papapanagiotou, I., and Yang, B. (2017). Internet of things: Survey on security and privacy. arXiv preprint arXiv:1707.01879.

Minoli, D., Sohraby, K., and Occhiogrosso, B. (2017). Iot considerations, requirements, and architectures for smart buildings-energy optimization and next-generation building management systems. IEEE Internet of Things Journal, 4(1):269-283.

Miorandi, D., Sicari, S., De Pellegrini, F., and Chlamtac, I. (2012). Internet of things: Vision, applications and research challenges. Ad hoc networks, 10(7):1497-1516.

Mumtaz, S., Alsohaily, A., Pang, Z., Rayes, A., Tsang, K. F., and Rodriguez, J. (2017). Massive internet of things for industrial applications: Addressing wireless iiot connectivity challenges and ecosystem fragmentation. IEEE Industrial Electronics Magazine, 11(1):28-33.

Qiu, T., Chen, N., Li, K., Atiquzzaman, M., and Zhao, W. (2018). How can heterogeneous internet of things build our future: A survey. IEEE Communications Surveys \& Tutorials, 20(3):2011-2027.

Sen, A. and Madria, S. (2017). Risk assessment in a sensor cloud framework using attack graphs. IEEE Transactions on Services Computing, 10(6):942-955.

Toulouse, M., Minh, B. Q., and Curtis, P. (2015). A consensus based network intrusion detection system. In 2015 5th International Conference on IT Convergence and Security (ICITCS), pages 1-6. IEEE.

UCI, C. (2013). Estatisticas de acesso web. https://archive.ics.uci.edu/ml/datasets/ Gas+Sensor+Array+Drift+Dataset. Acessado em 21/05/2018.

Wang, J., Liu, Z., Zhang, S., and Zhang, X. (2014). Defending collaborative false data injection attacks in wireless sensor networks. Information Sciences, 254:39-53.

Yang, L., Ding, C., Wu, M., and Wang, K. (2017). Robust detection of false data injection attacks for data aggregation in an internet of things-based environmental surveillance. Comp. Net., 129:410-428.

Yaqoob, I., Ahmed, E., ur Rehman, M. H., Ahmed, A. I. A., Al-garadi, M. A., Imran, M., and Guizani, M. (2017). The rise of ransomware and emerging security challenges in the internet of things. Computer Networks, 129:444-458.

Yu, Z. and Guan, Y. (2010). A dynamic en-route filtering scheme for data reporting in wireless sensor networks. IEEE/ACM Transactions on Networking (ToN), 18(1):150-163. 